\title{
Study on the Fiber Orientation during Compression Molding of Reinforced Thermoplastic Composites
}

\author{
Jin-Woo Kim' and Dong-Gi Lee,"\# \\ 1 Department of Mechanical System Engineering, Chosun University, 375 Seosuk-dong, Dong-gu, Gwangju, South Korea, 501-759 \\ \# Corresponding Author / E-mail: dglee@chosun.ac.kr, TEL: +82-62-230-7015, FAX: +82-62-233-7905
}

KEYWORDS: Short-fiber reinforced thermoplastic, Fiber orientation function, Fiber orientation angle, Compression ratio

\begin{abstract}
In this study, effect of fiber morphology on orientation is evaluated. Therefore, intersection angle for chopped strand, fiber length, and structure of fiber mat is changed to make the corresponding specimens and the relationship between compression ratio and fiber orientation is derived. Factors for controlling flow of glass fiber are glass fiber and matrix. 3 types of specimens, they are heated in oven as blank. Secondary hot press is applied. During compression molding, flow of test specimen is restricted to longitudinal direction. Measurement area for fiber orientation is $50 \mathrm{~mm} \times 50 \mathrm{~mm}$ area in length of molded part, and the image is processed to confirm fiber orientation function. Fiber orientation function value, which is an indicator for directionality of fiber, is increased along with longer fiber length and higher compression ratio. Higher needle punching number induces tangling of fibers, and less fiber orientation function can be achieved. Fewer needles punching number results the less interference between fibers and fiber orientation function is increased accordingly. Fiber orientation angle, an indicator for fiber directionality in composite, can be empirically estimated according to compression ratio and fiber morphology using the derived equations.
\end{abstract}

Manuscript received: June 27, 2014 / Revised: July 28, 2014 / Accepted: July 31, 2014

\section{Introduction}

Type of matrix in fiber reinforced plastics (FRP) can be divided into thermosetting resin and thermoplastic resin. According to matrix type, FRP can be classified as fiber reinforced thermosets (FRTS) and fiber reinforced thermoplastics (FRTP). Conventionally, FRP is a molded part with thermosetting resin. However, due to recycling, waste processing, and environment issue have been highlighted, and poor productivity of FRTS is the disadvantage to broad adoption. Recently, attention on glass fiber based FRTP using thermoplastic resin has been increased. This material features less specific gravity, and it is good for weigh reduction while maintaining the same performance. Because it is resin-based composite, it excels corrosion resistance and higher productivity. Also, thermoplastics can be cycled or incinerated easily.

Molding methods for fiber-reinforced plastic composites are impregnated molding and flow molding. Impregnation is applied to FRTS, and flow molding is adopted for FRTP, SMC, and Stampable sheet. Impregnation can increase fiber length and content compared with flow molding, and it features high strength and rigidity. However, productivity, work environment, and precise control of its dimension have the difficulty. On the contrary, flow molding performs better in productivity and precise dimension control. During flow molding, material will be flowed inside of mold, and thickness of molded part is easy to change. Therefore, high degree of freedom and complex shape of component can be molded. Due to these advantages, there are many researches conducted for high-temperature flow molding.

With this trend, fiber-reinforced plastic composite with glass fiber as reinforcement and thermoplastic resin as matrix receives the many attentions. Fiber-reinforced plastic composite has high strength, superior corrosion resistance, short molding process, and can save weight. Because of these advantages, it has been studied for aero plane, automobile, and other industries. ${ }^{1-4}$

The best way to achieve these goals could be engine efficiency improvement, new design of aerodynamic car body, and lightweight material. Especially, lightweight material can improve not only enhancing the performance of vehicle, but also improving fuel mileage. Therefore, it could be told that lightweight material is the most effective approach for preventing environment contamination and fuel saving. To solve these outstanding problems, researches and developments have been continuously conducted, and new material has been invented continuously. Materials that are used for structural member and machineries can be diverse, but the attention for 
composite materials has been significantly increased.

In the actual production environment for glass fiber-reinforced plastic composite, blank at about $190^{\circ} \mathrm{C}$ is put into mold at about 40 $80^{\circ} \mathrm{C}$, and it is pressed under about $15 \sim 20 \mathrm{MPa}$. After compression molding, it is quenched and demolded. As shown before, compression molding among the fabrication methods for glass fiber-reinforced plastic composites utilizes the heating up to melting point of matrix. At this temperature, fiber and matrix be separated due to different flow rate, and directionality of fiber due to displacement is occurred. Eventually, this cause anisotropy and inhomogeneity, which significantly influence mechanical properties. This composite material is affected by matrix, but the significant factor for mechanical properties is directionality of fiber reinforcement. ${ }^{5-8}$

After compression molding, mechanical properties of molded part are decided by fiber, and its corresponding flow behaviors, such as directionality and fiber content, should be reviewed according to configuration and molding conditions. According to the previous studies on the separation of fiber and matrix and fiber orientation, separation equation for fiber and matrix is derived based on solid-liquid two-phase flow theory, and concept of separation coefficient is implemented. In addition, fiber orientation is measured using image processing, and distribution of fiber content is analyzed accordingly. Researches on strength calculation for fiber-reinforced composite, influence of fiber content/orientation on tensile strength of GFRP composite, continuous manufacturing process for composite with impregnated thermoplastic into fiber mat including mathematical modelling, increasing impregnation level by measuring physical properties of polypropylene resin and glass fiber mat have been conducted. However, systematic study for impact of compression ratio on fiber orientation during compression molding of glass fiberreinforced plastic composite is not explored. ${ }^{9,10}$

In this study, influence of fiber morphology on orientation is evaluated. Therefore, intersection angle for chopped strand, fiber length, and structure of fiber mat is changed to make the corresponding specimens and the relationship between compression ratio and fiber orientation is derived.

\section{Theory}

\subsection{Compression Ratio}

After placing raw materials into two parallel plates like Fig. 1, flow to $y$ direction is limited, and material is compressed from initial thickness, $h_{0}$ to $h$. The corresponding compression ratio can be denoted as follows. ${ }^{11}$

$$
R_{c r}=1-\frac{h}{h_{0}}
$$

where, $h$ is thickness after molding, and $h_{0}$ is initial thickness.

If it is assumed that speed of composite is same as the one of matrix, average speed in $x$ direction can be calculated as follows.

$$
V_{a v}=\left(\frac{\dot{h}}{h}\right) X
$$

where, $\dot{h}$ is compression speed of press, and $h$ is thickness after molding.

\subsection{Fiber Orientation Function}

After placing raw materials into two parallel plates like Fig. 1, if flow direction to $y$ is limited during compression molding, fiber can be treated as 2-dimensional element because thickness of molded part be significantly smaller than width and length. The corresponding distribution of fiber orientation $(q(\theta))$ can be denoted as follows. ${ }^{12}$

$$
q(\theta)=\frac{1}{\pi}\left[\left(\frac{1-J}{1+J}\right) \cos ^{2} \theta+\left(\frac{1+J}{1-J}\right) \sin ^{2} \theta\right]^{-1}
$$

where, $J$ is fiber orientation function for 2-dimensional distribution. If fiber direction is isotropic, $J=0$. In case of uni-directional fiber, $J=1$. If fiber is rotated 90 degree from $J=1$ direction, it is $J=-1$.

Fig. 2 shows the relationship between fiber orientation angle in Eq. (3) and relative length of fiber. Where, relative length of fiber is the ratio of fiber for individual fiber orientation comparing with total length. In this picture, if $J=1$, linear relationship with principal orientation axis is exhibited. If $J=0$, it be perpendicular to principal

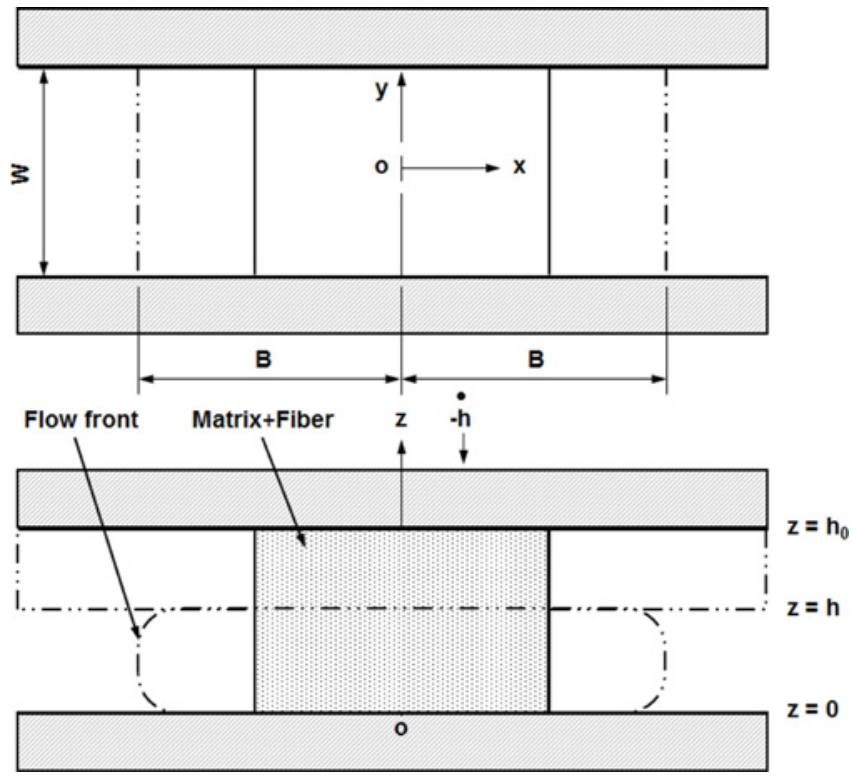

Fig. 1 Coordinate systems for compression mold slab-shaped part

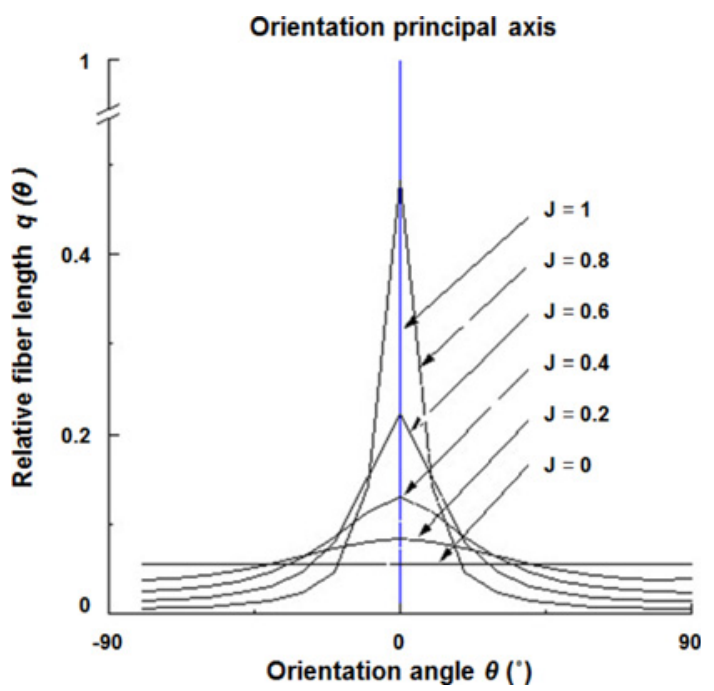

Fig. 2 Distribution of relative fiber length according to the fiber orientation angle 
orientation axis. While fiber orientation function reaches 0 , fiber shows isotropic behavior. For $J=1$, fiber becomes anisotropic.

\subsection{Equation for Fiber Orientation}

It only considers velocity incline in the direction of $x$ axis because of suppression the flow of $y$ direction in the Fig. $1 .^{13}$

In the Fig. 3, fiber with length $L$ positioned in the middle of the flow rotates by the difference of velocity in the direction of $x$ axis. Angular velocity of fiber is Eq. (4).

$$
\dot{\theta}=\left[\frac{V_{f a}-V_{f b}}{L} L\right] \sin \theta
$$

where, $V_{f a}$ and $V_{f b}$ are the velocity at both ends of fiber.

$$
V_{f a}=\frac{d V_{f}}{d x}\left(x+\frac{L}{2} \cos \theta\right) \quad V_{f b}=\frac{d V_{f}}{d x}\left(x-\frac{L}{2} \cos \theta\right)
$$

where, $d V_{f} / d x$ is velocity incline in the direction of $x$ axis.

Velocity difference at the both ends of fiber is

$$
V_{f a}-V_{f b}=\frac{d V_{f}}{d x} L \cos \theta
$$

Angular velocity of fiber $\theta$ from the Eq. (4) and (6) is

$$
\dot{\theta}=\frac{d V_{f}}{d x} \cos \theta \sin \theta
$$

In addition, in case separation of fiber positioned at the distance $x$ from the center of molding product occurs, velocity incline is

$$
\frac{d V_{f}}{d x}=\frac{\dot{h}}{h}-\frac{K_{s p} \gamma_{f} d_{f s}^{2}}{K_{m}}\left(1-n_{m}\right)\left(2 C \frac{\dot{h}}{h^{2}} X\right)^{-n_{m}}
$$

When substituting Eq. (8) with Eq. (7), angular velocity of fiber is

$$
\dot{\theta}=\left[\frac{\dot{h}}{h}-\frac{K_{s p} \gamma_{f} d_{f s}^{2}}{K_{m}}\left(1=n_{m}\right)\left(2 C \frac{\dot{h}}{h^{2}} X\right)^{-n_{m}}\right] \cos \theta \sin \theta
$$

\section{Experimental Methods}

During compression molding of fiber reinforced plastic composite (matrix: polypropylene, reinforcement: glass fiber), factors for controlling flow of glass fiber are glass fiber and matrix. To understand

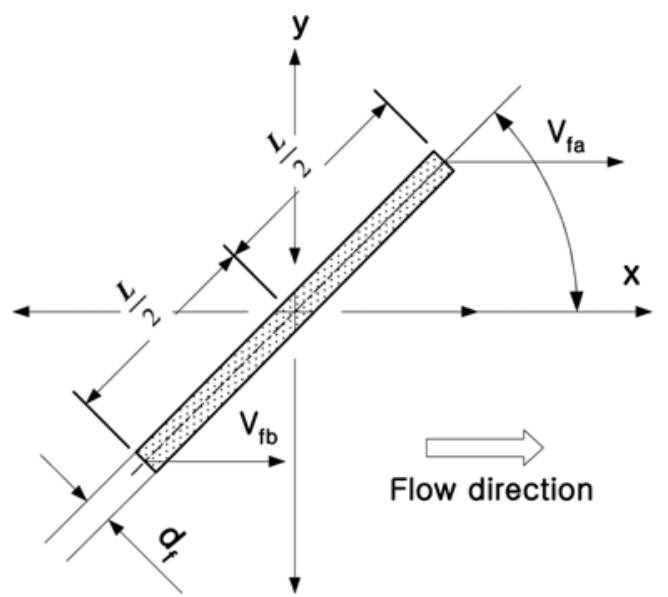

Fig. 3 Flow direction of fiber fiber orientation, 3 types of test specimens are prepared.

At the first experiment, $0.25 \mathrm{~mm}$ thick polypropylene sheet is cut into $60 \mathrm{~mm}$ (width) $\times 60 \mathrm{~mm}$ (length), and stacked for 18 sheets to make specimen. As shown in Fig. 4, intersection angle for chopped strand, $\theta_{0}$ is set to $30^{\circ}, 45^{\circ}$, and $60^{\circ}$ from $x$ direction. 3 locations in the middle of layers feature $13 \mathrm{~mm}$ of fiber intersection (strand tex: 75, filament diameter: $14 \mu \mathrm{m}$ ). In Fig. 5, the black part is glass fiber, the white one polypropylene. Also, compression ratio is 0.36 .

In second experiment, glass fiber is cut into $15 \mathrm{~mm}, 30 \mathrm{~mm}$, and $50 \mathrm{~mm}$ using roving cutter, and dispersed into air for stacking. It is made to glass fiber mat, which is stacked with matrix. After compression molding, the corresponding specimen is prepared. Fiber content $(w t \%)$ is set to $40 w t \%$, and $3.8 \mathrm{~mm}$ thick $\left(h_{0}\right) 3.8 \mathrm{~mm}$ of composite is fabricated. To measure, fiber orientation, prepared sheet is cut into $60 \mathrm{~mm}$ (width) $\times 60 \mathrm{~mm}$ (length) $\times 3.8 \mathrm{~mm}$ (thickness) by the length of reinforcement.

For third experiment, glass fiber is cut into $50 \mathrm{~mm}$ length using roving cutter, and it is dispersed into air for preparing stacked glass fiber mat, which is needle punched. In this case, needle punching number (Npn) is changed (10,20, and 50 times) to change structure of glass fiber mat. Diameter of needle is $1.753 \mathrm{~mm}$. Needle punch has triangular cross section, and there are 2 grooves per edge (total 6 grooves). Therefore, during needle punching of glass fiber mat, horizontally laid fiber is weaved into vertical direction. After stacking of glass fiber mat with matrix, hot press is used to fabricate test specimen $\left(h_{0}=3.8 \mathrm{~mm}, 40 w t \%\right)$.

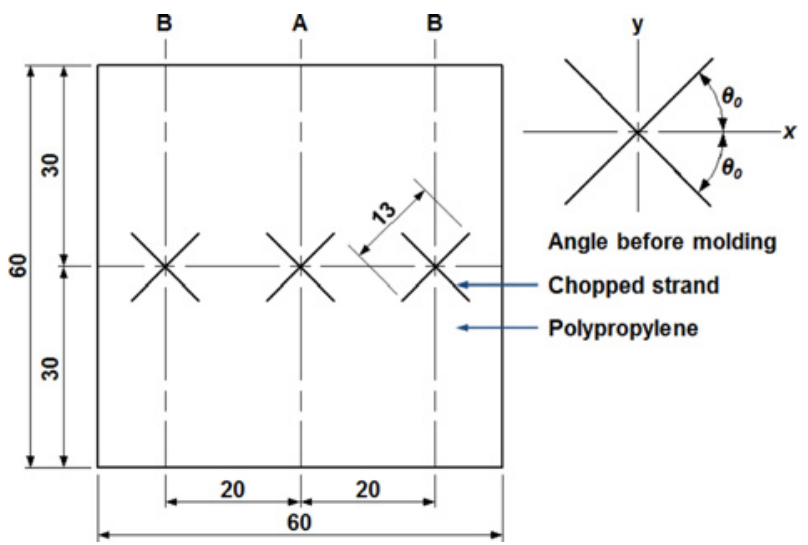

Fig. 4 Specimen for one-dimensional square shaped

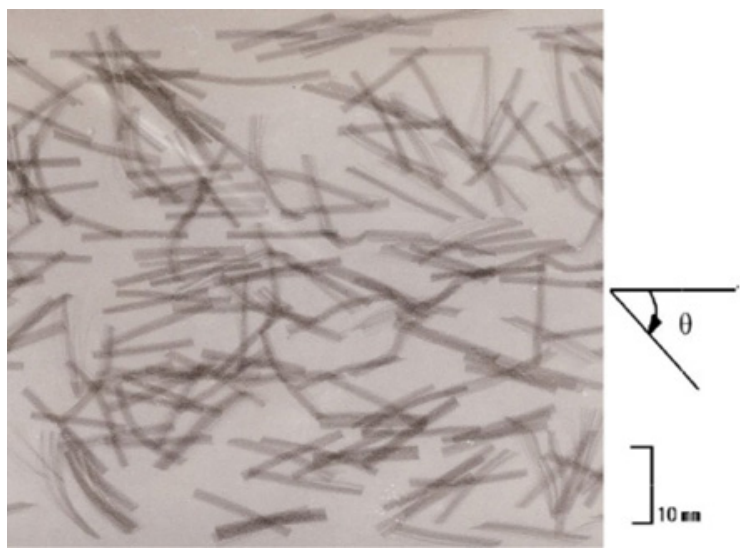

Fig. 5 Soft X-ray photograph $\left(R_{c r}=0.36\right)$ 
After preparing 3 types of specimens, they are heated in oven as blank. Secondary hot press is applied. In this time, dimension of mold cavity is $60 \mathrm{~mm} \times 240 \mathrm{~mm}$ with $190^{\circ} \mathrm{C}$ of blank temperature $\left(T_{B}\right), 60^{\circ} \mathrm{C}$ of mold temperature $\left(T_{M}\right), 25 \mathrm{~mm} \mathrm{~s}-1$ of compression speed for press. Compression tool is 30 -ton hot press. During compression molding, flow of test specimen is restricted to longitudinal direction as shown in Fig. 1.

Measurement area for fiber orientation is $50 \mathrm{~mm} \times 50 \mathrm{~mm}$ area from $B / 2$ point in length of molded part, and the image is processed to confirm fiber orientation function. Image scanner for process is Seiko Epson GT-4000V with $400 \times 400$ pixel, $1 / 8 \mathrm{~mm} \times 1 / 8 \mathrm{~mm}$ for 1 pixel, and 256 gray scales (gray level: black $=0$, white $=255$ ). For X-ray imaging, Mikasa Mammo Graphic System from Japan is used with Fuji film (MI-NC medical grade X-ray film, $180 \mathrm{~mm} \times 240 \mathrm{~mm}$ ).

\section{Results and Discussion}

To understand fiber orientation during compression molding of square plate, fiber intersection angle, $\theta_{0}$ and compression ratio is varied in test specimen. The corresponding test specimens are tested like Fig. 1. After hot press molding of test specimen with $\theta_{0}$ of intersection angle, fiber orientation angle of molded part is denoted as $\theta$. While, compression speed is $25 \mathrm{~mm} / \mathrm{s}$, and the compression ratios are 0.25 , $0.36,0.48,0.61$, and 0.73 .

On Fig. 6, fiber orientation of A and B section is shown. Center of molded part (section A) displays the less fiber orientation angle $(\theta)$ in Fig. 6, compared with section B. In addition, higher compression ratio induces higher flow rate, and bigger change in fiber orientation angle is found in section A and B. Average speed of A section is slower than the one for B section, and less orientation angle is exhibited. That is, negligible influence of speed gradient in $y$ direction elucidates the dominant influence of $\mathrm{x}$ direction in square plate.

Fig. 7 shows the relationship between compression ratio of molded part and fiber orientation according to fiber length $(L=15 \mathrm{~mm}, 30 \mathrm{~mm}$, and $50 \mathrm{~mm}$ ) where $40 w t \%$ of fiber content. From this figure, impact of compression ratio is higher than the one of fiber length. Because polypropylene matrix become liquid during compression molding, solid part, glass fiber, be affected significantly when fiber length is long according

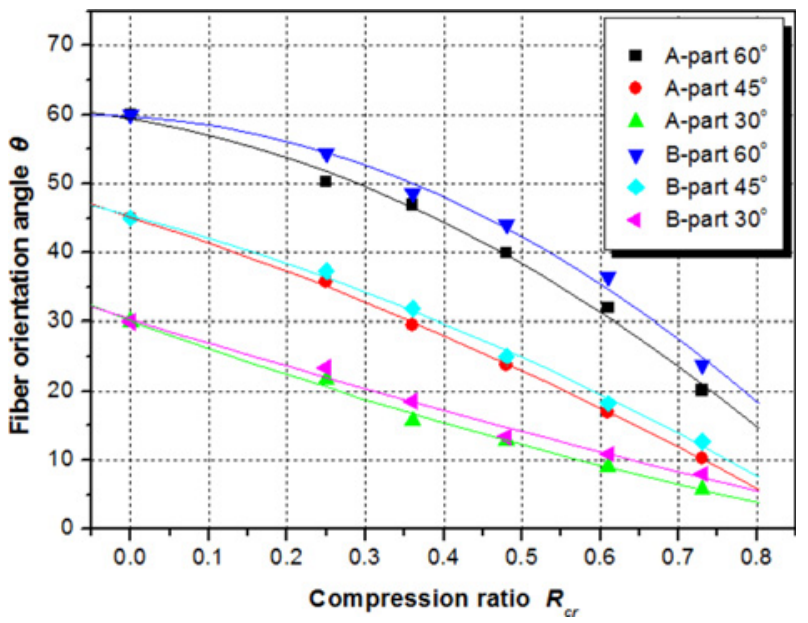

Fig. 6 Relationship between compression ratio $\left(R_{c r}\right)$ and fiber orientation angle $(\theta)$ to the difference in flow rate. In addition, longer fiber length in molded part shows the higher fiber orientation function value after compression molding. From the compression molding of glass fiber-reinforced plastic composite, relationship between fiber orientation function $(J)$ and compression ratio $\left(R_{c r}\right)$, which can be the indicator for directionality of fiber, can be empirically derived as Eq. (10), (11), and (12).

$$
\begin{aligned}
& J_{(L=50 \mathrm{~mm})}=0.111+\left(0.542 \times R_{c r}\right) \\
& J_{(L=30 \mathrm{~mm})}=0.085+\left(0.542 \times R_{c r}\right) \\
& J_{(L=15 \mathrm{~mm})}=0.057+\left(0.479 \times R_{c r}\right)
\end{aligned}
$$

Fig. 8 exhibits the relationship between fiber orientation function and compression ratio when structure of glass fiber mat is changed via needle punching. Higher compression ratio induces bigger deformation in molded part, and fiber orientation function is increased accordingly. Test specimen with larger needle punching count (Npn) in $1 \mathrm{~cm}^{2}$ of glass fiber mat shows the less fiber orientation function value because of tangled fibers. Specimen with less punching count displays the higher fiber orientation function. Therefore, fiber orientation function is

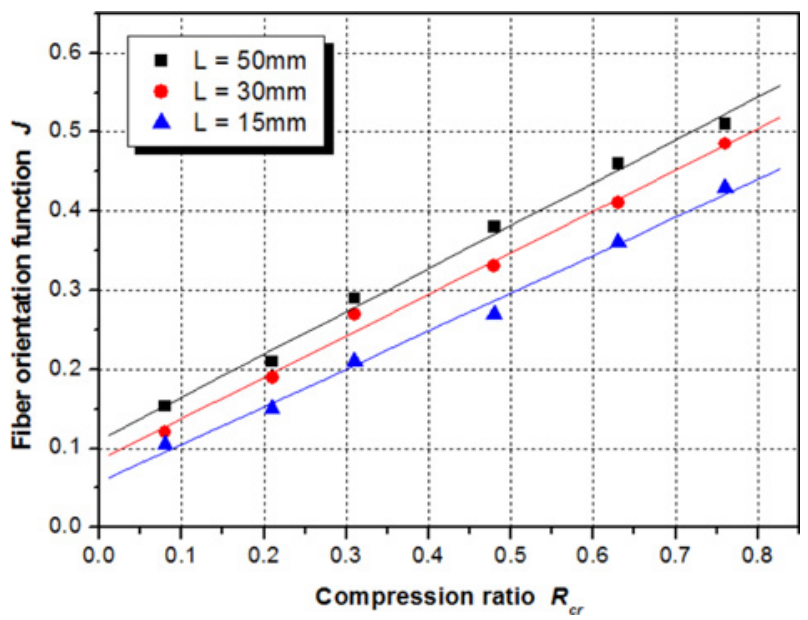

Fig. 7 Relationship between the compression ratio $\left(R_{c r}\right)$ and the fiber orientation function $(J)$ (fiber length)

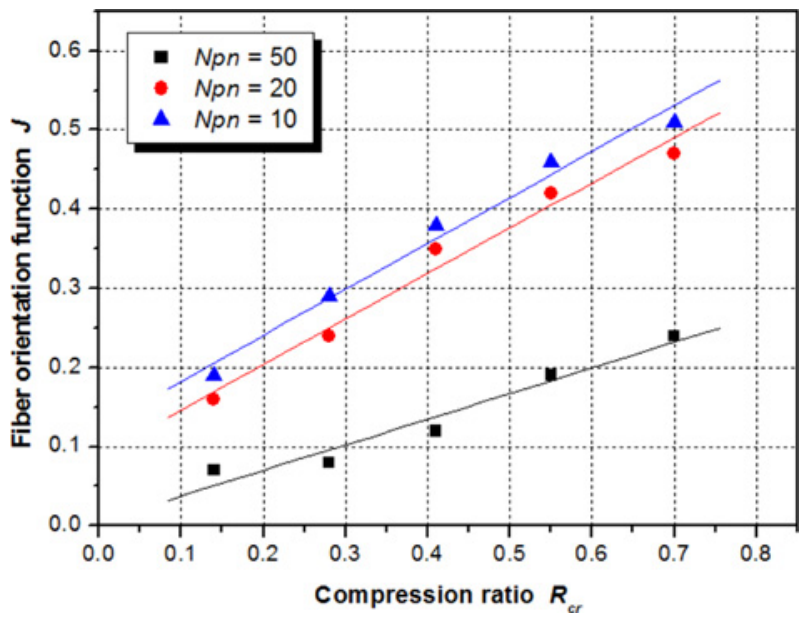

Fig. 8 Relationship between the compression ratio $\left(R_{c r}\right)$ and the fiber orientation function $(J)$ (needle punching count) 
affected by needle punching number and compression ratio. According to the test results with different needle punching numbers, relationship between fiber orientation function $(J)$ and compression ratio $\left(R_{c r}\right)$ can be determined as Eq. (13), (14), and (15), empirically.

$$
\begin{aligned}
& J_{(N p n=50)}=0.005+\left(0.324 \times R_{c r}\right) \\
& J_{(N p n=20)}=0.089+\left(0.573 \times R_{c r}\right) \\
& J_{(N p n=10)}=0.124+\left(0.581 \times R_{c r}\right)
\end{aligned}
$$

\section{Conclusions}

During compression molding of short-fiber reinforced plastic composite, factors of fiber morphology that can influence fiber orientation can be summarized as follows.

1) Intersection angle of chopped strand at the center of square plate be influenced minimally by speed gradient in $y$ direction, and gradient in $x$ direction dominates the influence on fiber orientation.

2) Fiber orientation function value, which is an indicator for directionality of fiber, is increased along with longer fiber length and higher compression ratio.

3) Higher needle punching number induces tangling of fibers, and less fiber orientation function can be achieved. Fewer needles punching number results the less interference between fibers and fiber orientation function is increased accordingly.

4) Fiber orientation angle, an indicator for fiber directionality in composite, can be empirically estimated according to compression ratio and fiber morphology using the derived equations.

\section{REFERENCES}

1. Ren, G., Hogg, P., and Woolstencraft, D., "Fire Reactions of Ceramic and Polymer Moulding Composites," Advances in Applied Ceramics, Vol. 109, No. 6, pp. 328-337, 2010.

2. Mei, H., "Modelling Environmental Effects on Stress Oxidation Behaviour of Carbon Fibre Reinforced Ceramic Matrix Composites," Advances in Applied Ceramics, Vol. 108, No. 2, pp. 123-127, 2009.

3. Hofmann, I., Haas, D., Eckert, A., Rüf, H., Firgo, H., et al., "Mechanical Properties of Cellulose-Apatite Composite Fibres for Biomedical Applications," Advances in Applied Ceramics, Vol. 107, No. 5, pp. 293-297, 2008.

4. Minay, E., Boccaccini, A., Veronesi, P., Cannillo, V., and Leonelli, C., "Sintering of Metal Fibre Reinforced Glass Matrix Composites using Microwave Radiation," Advances in Applied Ceramics, Vol. 104, No. 2, pp. 49-54, 2005.

5. Wang, Y., Zhou, J., and Zhang, S., "Construction of Real Digital Microstructures of Nanocrystalline Materials Considering Coupled Grain Size and Grain Orientation Distributions," Materials Research Innovations, Vol. 17, No. S1, pp. 115-118, 2013.
6. Park, J. H., Kim, T. W., Yim, H. J., and Lee, K. S., "Characterizations of the Interfacial Energy in Glass/Resin Laminates,” Int. J. Precis. Eng. Manuf., Vol. 12, No. 6, pp. 10791084, 2011.

7. Sait, A. N., "Optimization of Machining Parameters of GFRP Pipes using Evolutionary Techniques,” Int. J. Precis. Eng. Manuf., Vol. 11, No. 6, pp. 891-900, 2010.

8. Gencel, O., Ozel, C., Brostow, W., and Martínez-Barrera, G., "Mechanical Properties of Self-Compacting Concrete Reinforced with Polypropylene Fibres," Materials Research Innovations, Vol. 15, No. 3, pp. 216-225, 2011.

9. Kim, G., Hong, S., Kim, G. H., and Jhang, K.-Y., "Evaluation of Subsurface Defects in Fiber Glass Composite Plate using Lock-in Technique,” Int. J. Precis. Eng. Manuf., Vol. 13, No. 4, pp. 465-470, 2012.

10. Kim, J.-H., Shin, K.-B., and Kim, J.-S., "Optimum Design on Suspension Joint Parts of GFRP Composite Bogie Frame with HShaped Side Beams for Urban Railway Trains,” Int. J. Precis. Eng. Manuf., Vol. 13, No. 1, pp. 71-76, 2012.

11. Kim, J.-W. and Lee, D.-G., "Creep and Tensile Properties of Press Molding Joined Gmt-Sheets," Transactions of Nonferrous Metals Society of China, Vol. 21, No. S1, pp. s170-s174, 2011.

12. Kim, J.-W. and Lee, D.-G., "Effect of Fiber Orientation and Fiber Contents on the Tensile Strength in Fiber-Reinforced Composites," Journal of Nanoscience and Nanotechnology, Vol. 10, No. 5, pp. 3650-3653, 2010.

13. Kim, J., Kim, H., and Lee, D., "Study on Fibre Orientation of Weld Line Parts during Injection Moulding of Fibre Reinforced Plastic by Image Processing," Materials Research Innovations, Vol. 15, No. S1, pp. s303-s306, 2011. 